\title{
Modeling and Analysis of Population Dynamics of Examination Malpractice Among Students
}

\author{
${ }^{1}$ Obasi, Chinedu, ${ }^{2}$ Obiora, Collins, ${ }^{3} \mathrm{Mbah}$, Godwin C. E \& ${ }^{4}$ Olawuyi, Oluseye M \\ ${ }^{1,4}$ Department of Mathematics, Alvan Ikoku Federal College of Education. Owerri \\ ${ }^{2,3}$ Department of Mathematics, University of Nigeria, Nsukka \\ 1obasi1212@gmail.com; ${ }^{2}$ obiora.collins@unn.edu.ng; ${ }^{3}$ godwin.mbah@unn.edu.ng; and \\ ${ }^{4}$ mojirayooluseye@yahoo.com
}

\begin{abstract}
While examination malpractice has been a recognized social problem among students, the control of the phenomenon remains a challenge. In this study, we formulate a mathematical model describing the population dynamics of examination malpractice among students. Initial insight into the dynamics of the model is gained by analyzing some important mathematical features of the model such as the basic malpractice number. The malpractice-free equilibrium and endemic equilibrium points of the model are shown to be locally asymptotically stable when the basic malpractice number is less than unity. This result implies that examination malpractice can be totally eradicated among students when the basic malpractice number is less than unity. To understand the impact of controlling this social problem, we extend the model to incorporate awareness campaign and disciplinary measure as control strategies in curtailing the act. Our analysis reveals that incorporating control strategies have some influence in reducing examination malpractice among students. Further analysis indicates that considering both control strategies simultaneously yields a better result in reducing examination malpractice and examination malpractice will grow faster when control strategies are not introduced.
\end{abstract}

Keywords: Examination malpractice, stability analysis, basic malpractice number

\section{Introduction}

Examination malpractice is a social evil that can damage society to the extent of possibly leading to a failed state. It has very serious economic, political and social consequences. Examination malpractices render the goals of education invalid. The actualization of the goals of education will continue to be a mirage if the scourge of examination malpractice is not eradicated from the system. The country will end up producing graduates who lack the knowledge, skill and competence to exploit the resources of the nation. Besides, the graduates 
P a g e $\mid \mathbf{2}$

will lack the right type of values and attitude needed for survival in a globalized economy [1]. The fight against corruption cannot succeed if examination malpractice continues to be endemic in the educational system. This is so because examination malpractice is one of the vices or viruses that seem to have become entrenched in the country. It has become endemic and pandemic [5]. As leaders of tomorrow who have gone through a school system characterized by academic fraud and dishonesty, the youths of the country will sow and nurture this fraudulent behavior in any organization they find themselves. They will be destined to be a life of crime, fraud and corrupt practices [1].

Examination malpractice in the understanding of [2] is anything done by the examination candidate that is likely to render the assessment useless. Examination malpractice is, therefore, anything made by the stakeholders such as examination administrators, teachers, parents or students that is likely to render the assessment or examination ineffective or useless [2]. In the words of [5], examination malpractice is both contagious and infectious among students. It has polluted our academic environment and thus requires sanitary or sanitizing measures than punishment to get rid off [5]. It is a habit that can be acquired and also lost. It is also possible to resist examination malpractice in any form. It is thus similar in some respects to the epidemiology of diseases which have been modeled in many publications, yet it differs in other respect which makes it interesting to model. Examination malpractice has been as much in news as infectious diseases and should be accorded as much mathematical attention by way of modeling.

\section{Model Formulation}

Examination malpractice divides a school population in which it is active into three compartments namely: Susceptible-those who have not indulged in examination malpractice but can be involved if sufficiently tempted by friends, peers, etc. infective - those who are actively involved in examination malpractice. Resistant - those who do not involve themselves in examination malpractice, no matter what. Everyone is born susceptible. We assume a low interaction rate. Let $S(t), I(t), R(t)$, be the respective sizes at time $\mathrm{t}$, of the Susceptible, Infective and Resistant. The flow diagram is shown below.

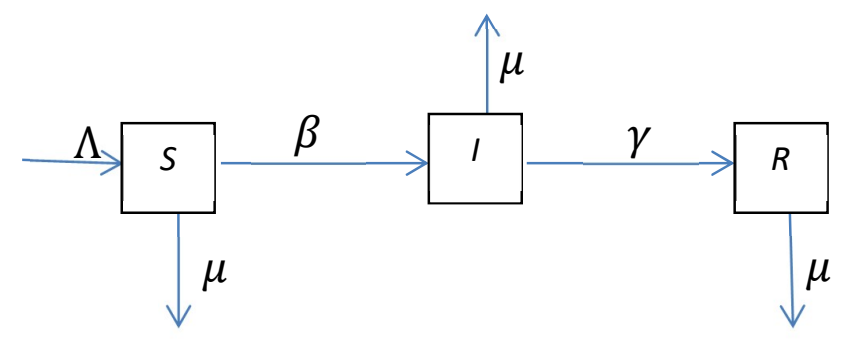


Table 1: The parameters of the model

\begin{tabular}{cl}
\hline Symbol & Description \\
\hline $\boldsymbol{\Lambda}$ & Admission rate into the school \\
$\boldsymbol{\beta}$ & Contact rate of $S$-class with $I$-class \\
$\boldsymbol{\mu}$ & Graduation rate from the school \\
$\boldsymbol{\gamma}$ & Rate at which students migrate from $I$-class to $R$-class \\
\hline
\end{tabular}

From the assumptions and the flow diagram together lead to the set of ordinary differential equations that describe the epidemiology of examination malpractice among Nigerian students:

$$
\begin{aligned}
& \frac{d S}{d t}=\Lambda-\beta S I-\mu S \\
& \frac{d I}{d t}=\beta S I-\mu I-\gamma \\
& \frac{d R}{d t}=\gamma I-\mu R
\end{aligned}
$$

The model under consideration monitors a human population as such; we assume that all the variables and parameters of the model are positive for all $t \geq 0$.

\section{Model Analysis}

In this section, we analyze the examination malpractice model (1) and investigate the existence and stability of its equilibrium points.

\subsection{Existence and Stability of Equilibrium Points}

We shall consider the cases below

Case 1: $\left(S^{0}, I^{0}, R^{0}\right)=\left(\frac{\Lambda}{\mu}, 0,0\right)$

Case 2: $\left(S^{*}, I^{*}, R^{*}\right)=\left(\frac{\mu+\gamma}{\beta}, \frac{\Lambda-\mu(\mu+\gamma)}{\beta(\mu+\gamma)}, \frac{\gamma}{\mu}\left(\frac{\Lambda \beta-\mu(\mu+\gamma)}{\beta(\mu+\gamma)}\right)\right)$ 


\subsection{The Basic Malpractice Reproduction Number, $\boldsymbol{R}_{0}$}

Model (1) has a unique malpractice-free equilibrium (MFE) given by $\left(S^{0}, I^{0}, R^{0}\right)=\left(\frac{\Lambda}{\mu}, 0,0\right)$. Thus, the basic malpractice reproduction number is given by

$$
R_{0}=\frac{\beta \Lambda}{\mu(\mu+\gamma)}
$$

\subsection{Local Stability of the Malpractice-Free Equilibrium Point $\boldsymbol{E}_{0}$}

The local stability of the MFE, $E_{0}=\left(\frac{\Lambda}{\mu}, 0,0\right)$ can be discussed by examining the linearized form of the model (1) at the given steady state $E_{0}$. This is done by computing the Jacobian matrix of the model (1). Linearizing (1) we obtain the following Jacobian matrix

$$
J=\left[\begin{array}{ccc}
-\beta I^{0}-\mu & -\beta S^{0} & 0 \\
\beta I^{0} & \beta S^{0}-\mu-\gamma & 0 \\
0 & \gamma & -\mu
\end{array}\right]
$$

Evaluating the Jacobian matrix at $E_{0}$, we get

$$
J_{0}=\left[\begin{array}{ccc}
-\mu & -\frac{\beta \Lambda}{\mu} & 0 \\
0 & (\mu+\gamma)\left(R_{0}-1\right) & 0 \\
0 & \gamma & -\mu
\end{array}\right]
$$

Now consider the matrix and let $\lambda$ be the eigenvalue. Then, we have $\left|J_{0}-\lambda I\right|=0$, where $J_{0}$ is the matrix above and $I$ is a $3 \times 3$ identity matrix. Thus, we have

$$
\left|J_{0}-\lambda I\right|=\left[\begin{array}{ccc}
-\mu-\lambda & -\frac{\beta \Lambda}{\mu} & 0 \\
0 & (\mu+\gamma)\left(R_{0}-1\right)-\lambda & 0 \\
0 & \gamma & -\mu-\lambda
\end{array}\right]=0
$$

This gives the characteristic equation

$(-\mu-\lambda)\left((\mu+\gamma)\left(R_{0}-1\right)-\lambda\right)(-\mu-\lambda)=0$

$\Rightarrow \lambda_{1}=\lambda_{2}=-\mu, \lambda_{3}=(\mu+\gamma)\left(R_{0}-1\right)$. For $\lambda_{3}$ to be negative, then $(\mu+\gamma)\left(R_{0}-1\right)<0 \Rightarrow$ $R_{0}-1<0$, since $\mu+\gamma>0$

$\therefore R_{0}<1$ 
Hence, all the eigenvalues have negative real parts if $R_{0}<1$, thereby making $E_{0}$ locally asymptotically stable.

\subsection{Local Stability of the Endemic Equilibrium Point $E_{1}$}

Evaluating the Jacobian matrix at $E_{1}$, we get

$$
J=\left[\begin{array}{ccc}
-\beta I^{*}-\mu & -\beta S^{*} & 0 \\
\beta I^{*} & \beta S^{*}-(\mu+\gamma) & 0 \\
0 & \gamma & -\mu
\end{array}\right]
$$

This gives the characteristic equation and eigenvalues

$\left(-\beta I^{*}-\mu-\lambda\right)(-\mu-\lambda)\left(\beta S^{*}-(\mu+\gamma)-\lambda+\beta^{2} S^{*} I^{*}\right)=0$

$\Rightarrow \lambda_{1}=-\mu, \lambda_{2}=-\left(\beta I^{*}+\mu\right)$ and $\lambda_{3}=\beta S^{*}-(\mu+\gamma)+\beta^{2} S^{*} I^{*}$. Then substituting the values of $S^{*}$ and $I^{*}$ at $E_{1}$ we observe that $\lambda_{2}=\frac{\mu(\mu+\gamma)-\Lambda \beta}{\mu+\gamma}-\mu$ is negative when $\mu(\mu+\gamma)-$ $\Lambda \beta<0$

$\Rightarrow \mu(\mu+\gamma)\left[1-\frac{\Lambda \beta}{\mu(\mu+\gamma)}\right]<0 \Rightarrow \mu(\mu+\gamma)\left(1-R_{0}\right)<0$

$\Rightarrow 1-R_{0}<0 \Rightarrow R_{0}>1$

Hence, $E_{1}$ is locally asymptotically stable when $R_{0}>1$.

\section{Control Model for Examination Malpractice}

In Nigeria today, examination malpractice is by far more widespread than HIV/AIDS. HIV/AIDS denies manpower to society, but examination malpractice provides ruinous manpower to society [3]. According to [3], the drugs to cure HIV/AIDS have not yet been discovered, but what is needed to cure examination malpractice is only social re-engineering. Therefore, the spread of examination malpractice can be reduced by introducing disciplinary measures to offenders and awareness campaign. In this section we explore the effect of these control measures by introducing them into the SIR model and performing dynamical system analysis of the new model. Let $D(t), A(t)$, be the respective sizes at time $\mathrm{t}$, of the disciplinary measure class and awareness campaign class. Hence, the new model becomes: 
$\frac{d S}{d t}=\Lambda-\beta S I-\mu S-\phi S$

$\frac{d A}{d t}=\phi S-(1-\epsilon) \beta A I-\mu A$

$\frac{d I}{d t}=\beta S I-(\mu+\gamma) I-\tau I$

$\frac{d D}{d t}=\tau I-(\xi+\mu) D$

$\frac{d R}{d t}=\gamma I+\xi D-\mu R$

where, $\phi:$ rate of the awareness campaign, $\epsilon$ : efficacy of the awareness campaign, $\tau$ : rate at which infective are being disciplined and $\xi$ : rate at which $D$ repent from the act as a result of disciplinary measures.

\subsection{Existence and Stability of the Equilibrium Point, $E_{0}$}

We shall consider the cases below

Case1: $\left(S^{0}, A^{0}, I^{0}, D^{0}, R^{0}\right)=\left(\frac{\Lambda}{\mu+\phi}, 0,0,0,0\right)$

\section{Case 2:}

$$
\begin{aligned}
& \left(S^{*}, A^{*}, I^{*}, D^{*}, R^{*}\right) \\
& =\left[\begin{array}{c}
\frac{\mu+\gamma+\tau}{\beta}, \frac{\phi(\mu+\gamma+\tau)}{\beta(1-\epsilon)(\mu+\phi)\left(R_{e}-1\right)-\beta \mu}, \frac{(\mu+\phi)\left(R_{e}-1\right)}{\beta}, \frac{\tau(\mu+\phi)\left(R_{e}-1\right)}{\beta(\xi+\mu)} \\
\frac{(\mu+\phi)\left(R_{e}-1\right)}{\mu \beta}\left(\gamma+\frac{\xi \tau}{\xi+\mu}\right)
\end{array}\right]
\end{aligned}
$$

\subsection{The Effective Malpractice Number, $R_{e}$}

We compute the effective malpractice number of the new model using the next generation matrix approach of [4]. The associated next generation matrices are:

$$
F=\left(\begin{array}{cc}
\beta S^{0} & 0 \\
0 & 0
\end{array}\right) \text { and } V=\left(\begin{array}{cc}
\mu+\gamma+\tau & 0 \\
-\tau & \xi+\mu
\end{array}\right) \text {.Then } F V^{-1}=\left(\begin{array}{cc}
(\xi+\mu) \beta S^{0} & 0 \\
0 & 0
\end{array}\right) \frac{1}{(\xi+\mu)(\mu+\gamma+\tau)}
$$

The effective malpractice number $R_{e}$ is the dominant eigenvalue of the matrix $F V^{-1}$ and is given: 


$$
R_{e}=\frac{\beta \Lambda}{(\mu+\phi)(\mu+\gamma+\tau)}
$$

\subsection{Local Stability of Malpractice Free Equilibrium Point for Control Model}

Theorem: The MFE of the model (4), is locally asymptotically stable (LAS) if $R_{e}<1$ and unstable if $R_{e}>1$, where $R_{e}$ has been defined above.

\section{Proof:}

By Theorem 2 in [4], the MFE is locally asymptotically stable (LAS) whenever $R_{e}<1$ but unstable if $R_{e}>1$. Therefore, we do not need to show the LAS of the MFE by the method of linearization of the system (at the MFE). Calculating $R_{e}$ using the method of the next generation matrix approach in [4] automatically proves the LAS of the MFE. Therefore, $E_{0}$ is locally asymptotically stable. This means that the number of examination malpractice cases will reduce and return to zero if $R_{e}<1$.

Based on the result, the effective malpractice number $R_{e}$ when awareness campaign and disciplinary measure are introduced into the SIR model is less than the basic malpractice number $R_{0}$, when there is no control measure in the model. That is,

$$
R_{e}=\frac{\beta \Lambda}{(\mu+\phi)(\mu+\gamma+\tau)}<\frac{\beta \Lambda}{\mu(\mu+\gamma)}=R_{0}
$$

This implies that the rate of spread of examination malpractice will be higher when there is no control measure than when awareness campaign and disciplinary measure are introduced in the school. Hence, awareness campaign and disciplinary measure reduce the rate of spread of examination malpractice.

\subsection{Local Stability of the Endemic Equilibrium Point $E_{1}$}

The Jacobian of the system of equations for the case 2 above is given below

$$
\stackrel{J_{0}}{=}\left[\begin{array}{ccccc}
-(\mu+\phi) & 0 & -\beta S^{0} & 0 & 0 \\
\phi & -\mu & 0 & 0 & 0 \\
0 & 0 & \beta S^{0}-(\mu+\gamma)-\tau & 0 & 0 \\
0 & 0 & \tau & -(\xi+\mu) & 0 \\
0 & 0 & \gamma & \xi & -\mu
\end{array}\right]
$$


The characteristic equation and the eigenvalues of the matrix above is given by

$$
\begin{aligned}
& \begin{array}{l}
\left(-\beta I^{*}-(\mu+\phi)\right. \\
\quad-\lambda)\left[\left(-(1-\epsilon) \beta I^{*}-\mu-\lambda\right)(-(\mu+\gamma+\tau)-\lambda)(-(\xi+\mu)-\lambda)(-\mu-\lambda)\right] \\
=0
\end{array} \\
& \begin{aligned}
\Rightarrow \lambda_{1}=-\left(\beta I^{*}\right. & +(\mu+\phi)), \lambda_{2}=-(\xi+\mu), \lambda_{3}=-\mu, \lambda_{4}=-(\mu+\gamma+\tau), \lambda_{5} \\
& =-(1-\epsilon) \beta I^{*}-\mu
\end{aligned} \\
& \therefore \lambda_{1}<0 \Rightarrow I^{*}>0 \Rightarrow \frac{(\mu+\phi)\left(R_{e}-1\right)}{\beta}>0 \Rightarrow R_{e}-1>0 \Rightarrow R_{e}>1
\end{aligned}
$$

Therefore, $E_{1}$ is locally asymptotically stable when $R_{e}>1$.

\section{Malpractice Growth Rate for the Models}

We can determine the rate at which this examination malpractice grows. The positive (dominant) eigenvalue of the Jacobian at MFE is typically referred to as the malpractice growth rate [4]. The Jacobian matrix of SIR model evaluated at the MFE is given by

$$
J_{0}=\left[\begin{array}{ccc}
-\mu & -\frac{\beta \Lambda}{\mu} & 0 \\
0 & (\mu+\gamma)\left(R_{0}-1\right) & 0 \\
0 & \gamma & -\mu
\end{array}\right]
$$

The Jacobian matrix has two distinct eigenvalues given by

$\lambda_{1}=\lambda_{2}=-\mu, \lambda_{3}=(\mu+\gamma)\left(R_{0}-1\right)$

We can easily observe that $\lambda_{1}, \lambda_{2}$ are negative, while $\lambda_{3}$ will be positive whenever $R_{0}>1$. Thus, the malpractice growth rate of the entire population is given by $\lambda^{*}=\lambda_{3}$. We observe from the above equations that if $R_{0}=1$, then the malpractice growth rate vanishes. On the other hand, if $R_{o}<1$, all the eigenvalues have negative real parts. This confirms that the MFE is locally asymptotically stable if $R_{o}<1$. Thus, a malpractice reproduction number greater than unity will result in an outbreak. Similarly, for the control model, the Jacobian matrix has four distinct eigenvalues given by

$$
\lambda_{1}=-(\mu+\phi), \lambda_{2}=\lambda_{3}=-\mu, \lambda_{4}=-(\xi+\mu) \text { and } \lambda_{5}=(\mu+\gamma+\tau)\left(R_{e}-1\right)
$$

We observe that $\lambda_{5}$ will be positive whenever $R_{e}<1$. Thus, the malpractice growth rate for the control model is given by $\lambda_{c}^{*}=\lambda_{5}$. Comparing the malpractice growth rates for the models, 
we observe that $\lambda^{*}-\lambda_{c}^{*}>0 \Rightarrow \lambda^{*}>\lambda_{c}^{*}$. This implies that the examination malpractice will grow faster when control strategies are not introduced.

\section{Conclusion}

A basic mathematical model describing the epidemiology of examination malpractice among Nigerian students is presented as a system of ordinary differential equations that we extend to incorporate awareness campaign and disciplinary measure as control strategies in curtailing the act. The malpractice-free equilibrium points are shown to be locally asymptotically stable, using the next generation matrix approach. The results of the analysis indicate that the examination malpractice epidemic can be reduced when both interventions, awareness campaign and disciplinary measure, are implemented. This result is shown by comparing the basic $\left(R_{0}\right)$ and effective $\left(R_{e}\right)$ malpractice numbers. Thus, the control strategies such as awareness campaign and disciplinary measure applied continuously can diminish $R_{e}<1$ and prevent the spread. Also, further analyses show that a malpractice number greater than unity will result in an outbreak. Based on this study, we conclude that both awareness campaign and disciplinary measure should be simultaneously applied to reduce the spread of examination malpractice among students. 


\section{References}

[1] Olatunbosun, J. B (2009). Examination malpractice in secondary schools in Nigeria: What sustains it? European Journal of Educational Studies 1(3), 101-108.

[2] Ojonemi, P.S., Enejoh, A \& Olatunmibi, O (2013). Examination malpractice: Challenges to human resource development in Nigeria. International Journal of Capacity Building in Education and Management (IJCBEM), Vol. 2 (1), 91-101.

[3] Opata, D.U (2003). Examination malpractice in Nigeria: Speaking frankly to our children and us. A Publication of the Centre for Child Development and Rehabilitation.

[4] P. Van den Driessche \& J. Watmough (2002). Reproduction numbers and sub-threshold endemic equilibrium for compartmental models of disease transmission. Math. Biosci. 180: 2948.

[5] Ibemesi, J.A (2007). From studies in polymer and vegetable oils to sanitization of the academic system. An Inaugural Lecture of the University of Nigeria, Nsukka. 